Provided for non-commercial research and education use. Not for reproduction, distribution or commercial use.

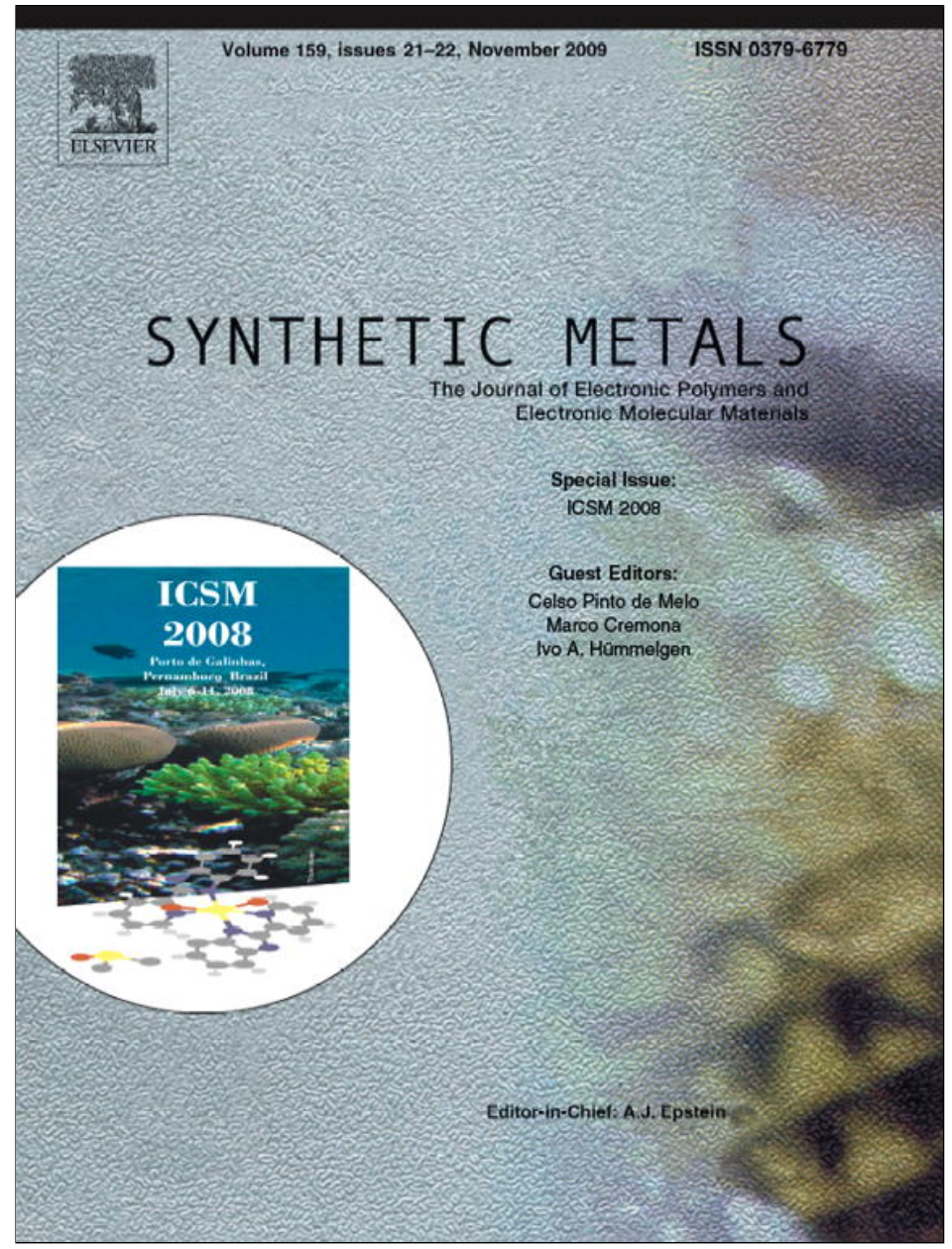

This article appeared in a journal published by Elsevier. The attached copy is furnished to the author for internal non-commercial research and education use, including for instruction at the authors institution and sharing with colleagues.

Other uses, including reproduction and distribution, or selling or licensing copies, or posting to personal, institutional or third party websites are prohibited.

In most cases authors are permitted to post their version of the article (e.g. in Word or Tex form) to their personal website or institutional repository. Authors requiring further information regarding Elsevier's archiving and manuscript policies are encouraged to visit:

http://www.elsevier.com/copyright 


\title{
Macroscopic-scale carbon nanotube alignment via self-assembly in lyotropic liquid crystals
}

\author{
Stefan Schymura ${ }^{\mathrm{a}}$, Eva Enz ${ }^{\mathrm{a}}$, Siegmar Roth ${ }^{\mathrm{b}}$, Giusy Scalia ${ }^{\mathrm{b}, \mathrm{c}}$, Jan P.F. Lagerwall ${ }^{\mathrm{a}, *}$ \\ a Martin Luther University Halle-Wittenberg, Institute for Chemistry - Physical Chemistry, Mühlpforte 1, 06108 Halle, Germany \\ ${ }^{\mathrm{b}}$ Max Planck Institute for Solid State Research, Heisenbergstrasse 1, 70569 Stuttgart, Germany \\ ${ }^{\mathrm{c}}$ ENEA CR Portici, 8055 Portici, NA, Italy
}

\section{A R T I C L E I N F O}

\section{Article history:}

Received 14 August 2008

Received in revised form 6 August 2009

Accepted 12 August 2009

Available online 9 September 2009

Keywords:

Carbon nanotubes

Lyotropic liquid crystal

Alignment

\begin{abstract}
A B S T R A C T
By dispersing carbon nanotubes (CNTs) in a lyotropic liquid crystalline matrix, uniaxial alignment of the nanotubes can easily be achieved over macroscopic areas. We briefly describe the principles behind the technique and then show that it can be applied to multiwall as well as single-wall nanotubes and that a variety of different dispersing materials can be used, from industrial surfactants to DNA. We also present a new microfluidics-based method for transferring the liquid crystal-dispersed CNTs to a substrate, maintaining a fair control of tube direction.
\end{abstract}

(c) 2009 Elsevier B.V. All rights reserved.

\section{Introduction}

Since their discovery in the early 1990s, carbon nanotubes (CNTs) have been recognized as a possible key component in a large range of new applications and materials, ranging from future nanoscale electronics devices to powerful filler materials in composites with enhanced mechanical and/or electronic properties [1-5]. The attractive properties of CNTs are largely due to their quasi-one-dimensional shape, with diameter in the nanometer range but length in the micron range. On the other hand, such extreme anisometry also makes control of the tube direction vital for many applications and a number of schemes have thus been developed for aligning CNTs, each of which has advantages and drawbacks and none of which is universally applicable [1,6-11]. Here we discuss the approach of dispersing the nanotubes in a liquid crystalline matrix $[6,10,12,13]$, a fluid exhibiting long-range orientational order, in some cases together with positional order. In fact, under the right conditions, CNTs can form liquid crystal phases on their own [14-17], just like any sufficiently concentrated suspension of sufficiently anisometric particles, as predicted by Onsager [18]. It is however highly challenging to reach acceptable CNT dispersion at the concentration required, and even if this threshold is reached, there is up until today no method known to obtain a large-scale uniform alignment of the sample. Therefore, suspension of CNTs in a host that is liquid crystalline in itself offers many practical advantages, as we will give examples in this

\footnotetext{
* Corresponding author. Tel.: +49 3455525 836; fax: +49 3455527400

E-mail address: jan.lagerwall@chemie.uni-halle.de (J.P.F. Lagerwall).
}

paper. If the CNTs are well dispersed they spontaneously align along the same direction as the host in order to minimize the free energy of the composite system. The liquid crystals used can be of thermotropic (the type used in displays) or lyotropic type, the latter being formed by amphiphilic molecules such as surfactants in water. Here we restrict ourselves to the latter class of liquid crystal.

We have previously shown that the liquid crystal phase used as the aligning host can be formed either from the same surfactant as used for dispersing the CNTs [19] or by a different surfactant, preferably with opposite charge to the CNT-dispersing surfactant [20]. The best results were obtained following the second route, the CNTs initially being dispersed in a low-concentration (about $1 \mathrm{wt} . \%$ ) solution of anionic sodium dodecyl benzene sulfonate (SDBS), with the liquid crystal phase being formed by the addition of cationic cetyl trimethyl ammonium bromide (CTAB) to a concentration of about 30 wt.\%. The negatively charged CNT-containing dodecyl benzene sulfonate micelles are most likely electrostatically complexed by the positively charged cetyl trimethyl ammonium micelles [20,21]. The resulting structure may be stabilized by the reduction in free energy due to the entropy increase when counter ions are released freely into solution [21]. In this way, CNT concentrations as high as $0.2 \mathrm{wt}$.\% could be uniformly dispersed and aligned by a hexagonal columnar liquid crystal host. The resulting suspension thereby gets the optical properties of a linear polarizer [20] since the nanotubes absorb light polarized along the tube axis. The dispersion can be stable over weeks or even months under optimized conditions. Even when these conditions are not met, stability over the time scale of several days is easily achieved, sufficient for the further processing of the aligned CNTs. 
Moreover, thin filaments can be pulled from the composite fluid, in which the CNTs are very well aligned along the filament direction, and these can be deposited on a substrate of choice. Followed by rinsing away the surfactant in water, this method provides a means of transferring the CNTs to target substrates with maintained control of nanotube orientation. While our previous work was done exclusively with single-wall carbon nanotubes (SWCNTs) dispersed using standard surfactants, we here extend the concept to multiwall tubes (MWCNTs) and to DNA-dispersed CNTs, and we present an additional method for transferring aligned tubes to substrates.

\section{Experimental}

\subsection{Dispersion of CNTS}

Dry CNT powder was weighed out in an Eppendorf vial which was subsequently filled with a low-concentration ( $\sim 1 \mathrm{wt} . \%)$ aqueous amphiphile (SDBS or single-stranded d(GT) 10 DNA [22]) solution. A high-power sonotrode (Dr. Hilscher) was then dipped into the vial and the system was sonicated typically for $30 \mathrm{~min}$, the vial being kept in an ice-water bath to counteract the considerable heating resulting from the sonication process. The success of the dispersion was verified in an optical microscope; the suspension was deemed useful for the further steps only if no bundling could be detected optically.

\subsection{Formation of liquid crystal phase}

Once a high-quality CNT dispersion had been obtained the amount of additional surfactant (here CTAB was used) required for forming a liquid crystal phase was added as a dry powder. After brief stirring, the sample was placed in a low-power sonication bath, where it was kept for $12-24 \mathrm{~h}$ in order to regain a homogeneous suspension.

\section{Results and discussion}

The lattice constant of the CTAB-based hexagonal columnar liquid crystal phase used as a host is in the order of a few nanometers [23], comparable to the effective outer diameter of a surfactantcovered SWCNT. While it is thus not surprising that SWCNTs can be well dispersed in the liquid crystal phase, it is not as obvious whether or not multiwall nanotubes, with diameters of several tens of nanometers, will be accepted. To investigate this issue, we dispersed purified MWCNTs with 10-30 nm outer diameter (SES Research, USA, >95\% CNTs vs. amorphous carbon, ash content $<0.2 \%$ ) using our standard method with SDBS as surfactant. CTAB was then added at the concentration required for the formation of a hexagonal columnar liquid crystal phase, yielding a final MWCNT concentration of $0.2 \mathrm{wt}$.\%. After homogenization the mixture was filled into an optically flat capillary for optical investigation.

Not only did the MWCNTs remain very well dispersed but also the mixture exhibited a distinct polarizer effect, cf. Fig. 1 (a: polarization parallel to liquid crystal alignment, b: polarization perpendicular), demonstrating that the MWCNTs had indeed been aligned by the liquid crystal host phase. The MWCNTs must certainly have induced at least a local distortion of the hexagonal lattice of the host phase, as their diameter (including surfactant sheath) is some 2-4 times the lattice parameter, but at the nanotube concentration studied here it may not have been severe enough to disrupt the phase structure. Another possibility is that the long-range hexagonal positional order of the phase is indeed disrupted by the MWCNT guests but not the long-range orientational order, i.e., the resulting phase would be nematic rather than
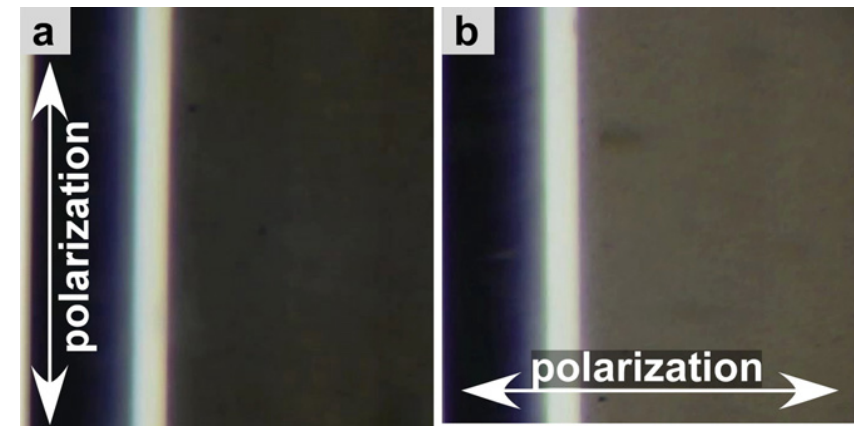

Fig. 1. Liquid crystal phase from CTAB in water with $0.2 \mathrm{wt} . \%$ SDBS-coated MWCNTs in a $200 \mu \mathrm{m}$ thick flat capillary (the bright vertical line in each photo is the left capillary edge; the CNT-liquid crystal mixture is to the right of this line). The liquid crystal is aligned along the capillary axis, i.e., vertically in the photos. The linear polarization of the illuminating light is indicated with arrows.

hexagonal columnar. The fact that the viscosity of the mixture is markedly lower than what is typical for hexagonal phases may suggest that this is the case. Further investigations of these issues are on-going.Next, we again studied the lyotropic liquid crystalinduced alignment of SWCNTs (purified HiPCO from CNI, USA, $<15 \mathrm{wt}$.\% ash content), but this time dispersed using d(GT) $)_{10}$ DNA rather than SDBS or SDS. DNA is also anionic, hence we expected DNA-dispersed CNTs to behave similar to SDBS-dispersed nanotubes. This is an interesting modification of our previous scheme, since DNA-dispersed CNTs can be fractionated according to their electronic properties [22]. Combined with our alignment technique, we can thus achieve aligned tubes of selected electronic type.

Upon addition of the dry CTAB powder, a precipitation of CNT aggregates was initially observed, but after homogenization in an ultrasound bath for some hours, the precipitate was easily redispersed and a quite homogeneous suspension was obtained. Most likely, the aggregates in the precipitate were not pure CNT bundles, which would not have been redispersed so easily in the relatively viscous liquid crystal environment, but rather CNTs inside DNA$\mathrm{CTAB}$ complex aggregates. These could be divided through the subsequent slow and gentle sonication process into small enough particles that most of them were no longer visible optically. They were then also well aligned by the liquid crystal host, as seen in Fig. 2. However, some relatively large-scale aggregates remained, and in these the CNT alignment was obviously random, as seen by the light intensity being unaffected by rotation of the polarization of incident light (see e.g., the dark aggregate at the bottom right of the photos in Fig. 2). It is our observation in general that highquality dispersion is a requirement for the alignment by the liquid crystal host phase to work. While the method is thus applicable also

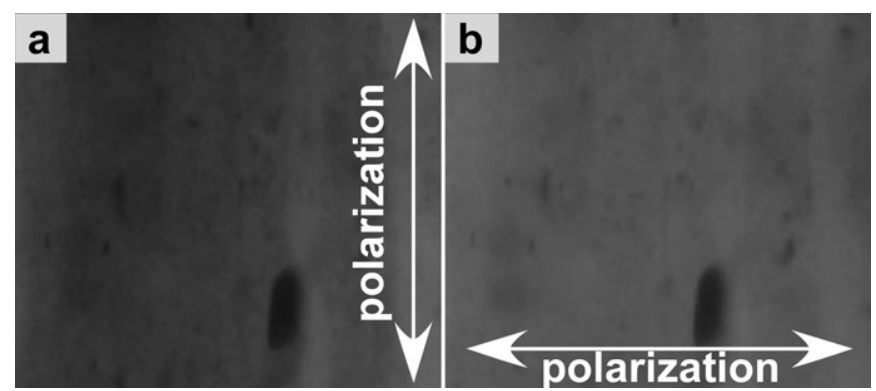

Fig. 2. Liquid crystal phase from CTAB in water with 0.2 wt.\% $d(G T)_{10}$ DNA-coated SWCNTs in a $200 \mu \mathrm{m}$ thick flat capillary, aligned parallel (a) and perpendicular (b) to the polarization of the illuminating light. Areas with non-uniform CNT distribution are either constantly dark or bright irrespective of illumination polarization, as seen on the right side of the sample. 


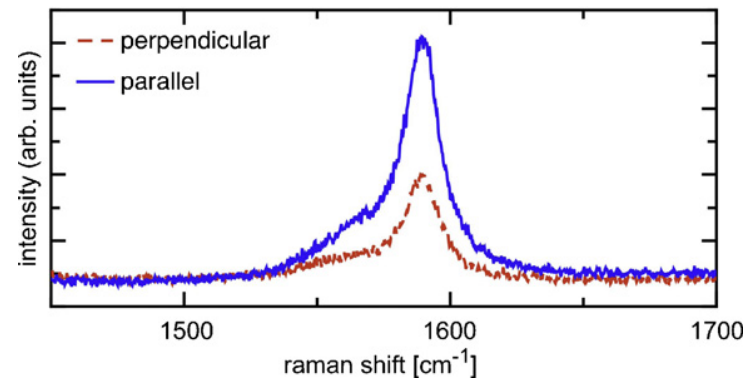

Fig. 3. G-band Raman scattering intensity from a line of SWCNT-liquid crystal mixture deposited on a double-sided scotch substrate, the surfactants having been gently rinsed away after deposition by immersion of the sample in water. The difference in intensity for polarization parallel and perpendicular to the line demonstrates that the CNTs are largely aligned along the direction of deposition.

to DNA-dispersed CNTs, further optimization of the experimental procedure is required to avoid aggregates in which the CNTs cannot be aligned.

Finally, as a new means of transferring liquid crystal-aligned CNTs to substrates, we developed a set-up where a CTAB-based liquid crystal mixture with $0.2 \mathrm{wt} . \%$ SDBS-dispersed SWCNTs was slowly pumped by a pressure-driven microfluidics device (Fluigent), allowing a controlled and continuous flow, through a tapered glass capillary. This acted as a 'pen' with which we could write thin lines of the suspension on a substrate of our choice by slowly moving the tip across the substrate. Because the liquid crystal was aligned along the capillary due to the flow (as verified by observing it between crossed polarizers), we expected that the alignment would be retained along the deposition direction also after the transfer to the substrate. We deposited lines in different directions on teflon bands as well as double-sided scotch tape, gently immersing the sample in water after deposition in order to rinse away the surfactant. After rinsing and drying, the samples were investigated with polarized Raman spectroscopy to verify if the CNTs were still on the substrate or had been rinsed away, and if they were aligned along the direction of deposition. SWCNTs have characteristic Raman modes that allow the presence of SWCNTs to be easily detected. Since the Raman intensity is dependent on the angle between nanotube axis and polarization of the excitation laser-the maximum occurring for parallel polarization-the orientation of the CNTs (or lack thereof) can also be easily verified using this technique [24].

The precise experimental conditions had strong impact on the end result, inadequate procedures resulting in loss of CNTs and/or loss of the alignment. With an appropriate deposition procedure followed by immediate rinsing in water for $10-20 \mathrm{~h}$, however, decent results were obtained, considering the simplicity of the procedure. Fig. 3 shows the intensity of the SWCNT Raman G-band of a line deposited horizontally, excited with light polarized vertically (red, dashed line) and horizontally (blue, continuous line). The difference in Raman scattering intensity indicates that the CNTs indeed largely maintained their alignment along the deposition direction. However, the contrast is not yet satisfactory and the smallest line thickness achieved was in the order of microns, hence these very first results are interesting only as an indication that the procedure may become useful in the future, once optimization of the experimental parameters and reduction of feature size has been achieved.

\section{Conclusions and outlook}

We have shown that the concept of aligning carbon nanotubes in lyotropic liquid crystals is quite versatile, being applicable to multiwall tubes with diameters considerably larger than the lattice constant of the native hexagonal columnar liquid crystal phase used as a host (possibly the phase in presence of CNTs is nematic), as well as for DNA-dispersed single-wall nanotubes. Moreover, the CNTs can be deposited onto substrates with a fair degree of retained alignment using a very simple writing procedure, where a CNT-liquid crystal suspension is gently pumped through a tapered capillary by a microfluidics device. While the optimization of this procedure is needed before a viable transfer technique is achieved, it provides an alternative route to our previous method based on the deposition of thin filaments pulled from the suspension [20].

\section{References}

[1] Y. Yan, M. Chan-park, Q. Zhang, Small 3 (2007) 24.

[2] M. Zhang, J. Li, Mater. Today 12 (2009) 12.

[3] R.H. Baughman, A.A. Zakhidov, W.A. de Heer, Science 297 (2002) 787.

[4] V. Sgobba, D.M. Guldi, J. Mater. Chem. 18 (2008) 153.

[5] W. Lu, C.M. Lieber, Nat. Mater. 6 (2007) 841.

[6] J.P.F. Lagerwall, G. Scalia, J. Mater. Chem. 18 (2008) 2890

[7] G.T. Liu, Y.C. Zhao, K. Deng, Z. Liu, W.G. Chu, J.R. Chen, Y.L. Yang, K.H. Zheng, H.B. Huang, W.J. Ma, L. Song, H.F. Yang, C.Z. Gu, G.H. Rao, C. Wang, S.S. Xie, L.F. Sun, Nano Lett. 8 (2008) 1071.

[8] S.J. Zhang, K.K.K. Koziol, I.A. Kinloch, A.H. Windle, Small 4 (2008) 1217.

[9] H.K. Bisoyi, S. Kumar, J. Mater. Chem. 18 (2008) 3032

[10] S. Kumar, H. Bisoyi, Angew. Chem. (Int. Ed.) 46 (2007) 1501.

[11] G.H. Yu, A.Y. Cao, C.M. Lieber, Nat. Nanotechnol. 2 (2007) 372.

[12] I. Dierking, G. Scalia, P. Morales, D. Leclere, Adv. Mater. 16 (2004) 865

[13] M. Lynch, D. Patrick, Nano Lett. 2 (2002) 1197.

[14] S.J. Zhang, S. Kumar, Small 4 (2008) 1270.

[15] P. Rai, R. Pinnick, A. Parra-vasquez, V. Davis, H. Schmidt, R. Hauge, R. Smalley, M. Pasquali, J. Am. Chem. Soc. 128 (2006) 591.

[16] S. Badaire, C. Zakri, M. Maugey, A. Derre, J.N. Barisci, G. Wallace, P. Poulin, Adv. Mater. 17 (2005) 1673.

[17] W.H. Song, I.A. Kinloch, A.H. Windle, Science 302 (2003) 1363.

[18] L. Onsager, Ann. N.Y. Acad. Sci. 51 (1949) 627.

[19] J.P.F. Lagerwall, G. Scalia, M. Haluska, U. Dettlaff-Weglikowska, S. Roth, F. Giesselmann, Adv. Mater. 19 (2007) 359.

[20] G. Scalia, C. von Bühler, C. Hägele, S. Roth, F. Giesselmann, J.P.F. Lagerwall, Soft Matter. 4 (2008) 570.

[21] C.R. Safinya, K. Ewert, A. Ahmad, H.M. Evans, U. Raviv, D.J. Needleman, A.J. Lin, N.L. Slack, C. George, C.E. Samuel, Philos. Transact. A: Math. Phys. Eng. Sci. 364 (2006) 2573.

[22] M.Zheng, A. Jagota, M.S. Strano, A.P. Santos, P. Barone, S.G. Chou, B.A. Diner, M.S. Dresselhaus, R.S. McLean, G.B. Onoa, G.G. Samsonidze, E.D. Semke, M. Usrey, D.J. Walls, Science 302 (2003) 1545.

[23] X. Auvray, T. Perche, C. Petipas, R. Anthore, M. Marti, I. Rico, A. Lattes, Langmuir 8 (1992) 2671.

[24] G. Duesberg, I. Loa, M. Burghard, K. Syassen, S. Roth, Phys. Rev. Lett. 85 (2000) 5436. 